\title{
Exploraciones en Fútbol y Redes Sociales Análisis del desempeño deportivo durante la Eurocopa 2004 a partir del análisis de redes sociales
}

\author{
Javier Bundio y Matías Conde - Universidad de Buenos Aires ${ }^{1}$
}

\begin{abstract}
Resumen
El presente trabajo propone una nueva forma de análisis del fútbol que se fundamenta en la perspectiva de redes sociales. Tomando como objeto redes construidas en base a los partidos de la última Eurocopa, se desarrollan una serie de construcciones que combinan el ARS tanto con los fundamentos tácticos y estratégicos como con la estadística clásica de este deporte, en la búsqueda de mostrar los valiosos aportes que pueden surgir de dicha conjunción. En este marco desplegamos, entre otras cuestiones, la posibilidad de mostrar la importancia de determinados vínculos dentro del campo de juego a partir de las propiedades de peso de los lazos, el virtual correlato entre la centralidad de determinados nodos y lo que se espera de jugadores tácticamente claves, y la interesante complementariedad que aparece entre la densidad de un componente y el porcentaje de posesión de balón que muestra un equipo.

Se incluyen también, una serie de aplicaciones posibles de este análisis, dentro del periodismo deportivo como en el campo también en el de asesoría técnica, a la vez que buscamos fundamentar la incursión del ARS en el fútbol no solo por su valor metodológico, sino también por su importancia didáctica e incluso ideológica.
\end{abstract}

Palabras clave: fútbol - estadística deportiva - análisis de tácticas - análisis de estrategias.

\begin{abstract}
This article presents a new way of soccer analysis based on social network perspectives. Taking some networks from the games of the last Eurocup as a study object, we develop some constructions which mix SNA with tactics and basic strategies and also with classical soccer statistics, trying to show the potential of the combined analysis.

In the first part we present some methodological possibilities (mix density levels from a component with ball-possesion from a team, centrality level form a node with tactical expected participation from a player, ties size and key relations in a team, and many others). In the second one, we offer some possible applications of this analysis in sport-press and as technical advisory for coaches. And finally we try to support that the combination between social network analysis and soccer is done not only with methodological reasons, but with didactical and ideological reasons.
\end{abstract}

Keywords: Soccer - sports statistics - tactical analysis - strategic analysis.

\section{I ntroducción: ¿Por qué aplicar el análisis de redes sociales al fútbol?}

\footnotetext{
${ }^{1}$ Enviar correspondencia a: arsfutbol@gmail.com
} 
Las redes sociales, como señala Steve Borgatti, son un campo de rápido crecimiento que se involucra cada vez más en distintas ramas, proponiendo una perspectiva que se enfoca sobre el desempeño grupal, ya que dentro de su marco, el valor social prevalece sobre el humano (2003: 29-31). Desde este enfoque es de donde parte nuestro interés por aplicar el análisis de redes sociales (en adelante ARS) al fútbol, entendiéndolo como deporte de equipo, donde el todo es más importante que la suma de las partes.

Sin embargo, no resulta sencillo plantear este tipo de propuesta. No solo porque el fútbol nunca fue un tópico con demasiada apertura en el ambiente científico (2004b: 1), sino porque al aplicarlo sobre esta disciplina, se corre el riesgo de caer en el uso y abuso del concepto de redes. $Y$ entendemos que una mala utilización de dicho concepto, no haría más que otorgar argumentos en favor de aquellos que encuentran en el ARS poco más que una moda pasajera tendiente a convertirse en una categoría vacía y sin sentido, tal advirtiera Ana Laura Rivoir en su artículo sobre los usos del concepto de redes (1999: 1).

Por esto, entendemos que vale la pena dejar en claro en el inicio mismo de este trabajo desde dónde lanzamos nuestros desarrollos (1999:13). Aquí no se propondrán aplicaciones con pretensiones revolucionarias que busquen cambiar completamente el modo de ver el fútbol a partir del análisis de redes, ni se enfatizará en las ventajas del ARS por sobre la estadística clásica de este deporte, ni mucho menos se buscará matematizar el fútbol a partir de una serie de algoritmos que el análisis nos provee. Y esto no sólo por el carácter exploratorio del trabajo aquí presente, sino porque creemos que los posibles aportes del ARS al fútbol apuntan hacia otra dirección. En otras palabras, impulsaremos expresamente la utilización de las redes sociales como instrumento metodológico por sobre su concepción como categoría sociológica (2004), aunque sin apelar a una rigurosidad que nos impida correlacionar el mismo con métodos cuantitativos y cualitativos provenientes de la propia ciencia de redes como de otras. Se verá que buena parte de este trabajo está orientada a la búsqueda de complementariedad entre desarrollos propios del ARS con conceptos provenientes del fútbol. 
¿Por qué creemos que esto es posible? Primero, porque el ARS nos provee de mútiples niveles de análisis (desde los actores a las estructuras pasando por díadas y grupos) (2003: 26), que favorecen el estudio de este deporte, donde lo individual y las pequeñas asociaciones pueden ser claves. A la vez que el equipo como un todo, aparece como la unidad más importante. Segundo porque esta aproximación al balompié desde una perspectiva de complejidad, entendiéndola como la aparición de propiedades emergentes provenientes de la interacción local de un número de componentes $\mathrm{y}$, considerando que el comportamiento complejo representa una situación intermedia entre un estado ordenado y uno desordenado (2007: 16-17), resulta particularmente interesante posicionarse desde este enfoque para analizar un deporte definido por uno de sus más grandes teóricos como la dinámica de lo impensado (1967). La ciencia de redes aplicada a un juego donde la lógica no siempre se da en tiempo y forma dando lugar a resultados imprevisibles y situaciones imprevistas en mayor cantidad que en cualquier otro deporte de equipo. Y además, dadas sus características, siempre ha sido esquivo a cualquier intento de formalización tradicional (especialmente aquellas que se importaban desde otras disciplinas como el básquet o el handball), convirtiéndose entonces en una cuestión particularmente atractiva para desarrollar un análisis de características contraintuitivas.

\section{Recolección, importación y mapeo de datos}

En el año 2004 el encuentro de dos entusiastas estudiosos españoles, José Luis Molina González y Juan Julián Merelo Cuervos ${ }^{2}$, interesados tanto en las redes sociales como en el fútbol (cuya relación se había originado a partir de la lista de correo de la revista REDES) ${ }^{3}$, dio lugar a un original juego de pronósticos deportivos que titularon "Redes contra Redes"4. El mismo estaba basado en la Eurocopa de fútbol $^{5}$ que, por ese entonces, estaba próxima a jugarse en Portugal. Para participar del juego había que enviar una predicción sobre quiénes serían los

\footnotetext{
2 J.J. Merelo Cuervos además de ser uno de los creadores del juego "Redes contra Redes", respondió amablemente todas nuestras consultas relativas a dicha competencia, siendo de gran ayuda a los fines del presente trabajo.

${ }^{3}$ Disponible en http://www.revista-redes.rediris.es

4 Tal intercambio (aún disponible en http://revista-redes.rediris.es/webredes/eurocopa/datos.htm) resultó uno de los disparadores más eficientes de temáticas desarrolladas en esl presente trabajo.

${ }^{5}$ La Eurocopa es el torneo de fútbol de selecciones más importante después de la Copa del Mundo. Juegan 16 equipos que clasifican a través de un sistema de eliminatorias del cual participan la totalidad de los equipos afiliados a la UEFA (la Unión Europea de Fútbol Asociado) que son más de 70. La ultima Euro se jugo en Portugal y fue ganada sorpresivamente (al punto de pagar 100-1 en las apuestas), por el seleccionado de Grecia, que baso su juego en un planteo defensivo y conservador que le dio excelentes resultados. Actualmente se están disputando las eliminatorias correspondientes al torneo de 2008.
} 
cuatro primeros del torneo, con la particularidad de que la misma debía fundamentarse (o al menos, una parte de ella) en el análisis reticular. En efecto, se pondrían a disposición de los participantes no solo las estadísticas típicas de este deporte (tiros al arco, posesión del balón, córners, etc), sino también y por primera vez, se proveería de datos reticulares, esto es, el detalle de los pases que los jugadores de un mismo equipo se daban entre sí.

Por suerte para los organizadores de dicho concurso, y aún cuando la discusión de cómo tomar dichos datos se ponía cada vez más interesante ${ }^{6}$, la empresa Benq recolectó por primera vez esta variante reticular como parte de su trabajo para el servicio de estadísticas de la Union of European Football Associations (UEFA) ${ }^{7}$. Aprovechando dicha situación, J. J. M. Guervos depuró los datos obtenidos y los puso a disposición de los participantes en ficheros de extensión “.DL" listos para ser descargados e interpretados a través del software correspondiente. Tal esfuerzo resultó muy útil tanto para quienes enviaron sus pronósticos (bastante errados por cierto) para dicha contienda, como para los fines de este trabajo, ya que no hemos podido dar con otra base de datos (más allá de la que estamos generando, como veremos más adelante, a partir de recursos propios) que tuvieran en cuenta los datos reticulares necesarios para hacer un análisis de redes sociales de un partido de fútbol tal como aquí se propone.

\section{Mapeo de la red}

El primer paso para mapear la red fue bajar los archivos ".DL" de las redes seleccionadas $^{8}$ e importarlos a través del UCINET. Tal importación daba como resultado una grilla de $23 \times 23$ (dado que 23 es el número de jugadores que conforman el plantel completo de una selección en una Eurocopa). Pero, como sabemos, en un partido de fútbol solo pueden participar entre 11 y 14 jugadores (teniendo en cuenta la posibilidad que tienen los técnicos de realizar hasta 3 cambios), por lo que nuestra red debía depurarse dejando de lado a los miembros del plantel que no tuvieran participación en el match. A tales fines recurrimos

\footnotetext{
6 La misma se encuentra disponible en el archivo de la Lista REDES, disponible en http://listserv.rediris.es/cgi-bin/wa?Al=ind0406\&L=redes

${ }^{7}$ La Union Europea de Fútbol Asociado, más conocida como UEFA, es la asociación que núclea a las entidades representantes del fútbol nacional de cada uno de los países de Europa (y desde 1994, por cuestiones políticas, también incluye a Israel), y representa al continente ante la Federación Internacional de Fútbol Asociado (FIFA).

${ }^{8}$ Dada la extensión y el tiempo de desarrollo de este trabajo se eligió trabajar solo con algunas de las redes disponibles. Basándonos en un criterio de relevancia y casos extremos se tomaron, de las 72 posibles, por un lado las redes de dos de los partidos más importantes (los choques entre Portugal y
} 
(guiados por los propios creadores del juego Redes contra Redes) a la función "No Isolates" (en el menú "Data" del Ucinet) que elimina todos aquellos nodos que no tienen relación ( $\mathrm{ni}$ de ida ni de vuelta) con los restantes. De esto deducimos, que en estas redes cada nodo equivale a un jugador (titular o suplente que ingresara al campo) que haya recibido o dado un pase a un compañero de equipo. Y justamente son los pases entre compañeros, los que hacen las veces de lazos ${ }^{9}$.

No obstante, el hecho de que el pase implique un emisor y un receptor supone la necesaria direccionalidad de los lazos. Una característica no menor y de notable importancia a la hora de desarrollar aportes desde el ARS al fútbol, ya que el hecho de que se tenga en cuenta desde y a quien va dirigida la pelota, permite profundizar el análisis de las acciones de los futbolistas. El periodismo muchas veces realiza un seguimiento especializado que llega a destacar no solo cuantos pases correctos fueron realizados por un jugador, sino que además incluye el detalle de qué pierna (o si fue con la cabeza) fue utilizada para realizar el pase. Pero no suele tomar en cuenta hacia qué jugador fue dirigido el pase. Un detalle no menor que, como veremos más adelante, muestra implicancias tácticas más que interesantes.

\section{Cuestión de peso: Granovetter al revés}

Como vemos en la Figura 1, trabajamos con redes no dicotomizadas que muestran una diferencia de peso importante entre algunos lazos, reflejando de este modo relaciones más fuertes entre determinados jugadores y ad hoc, a los fines de este trabajo, consideramos que los futbolistas respetaron sus posiciones durante todo el partido en determinados sectores del campo. Lo que nos permite esta cuestión es dirimir cuáles son las relaciones más importantes a partir de ir estableciendo umbrales de corte cada vez más altos.

\footnotetext{
Grecia que curiosamente fueron tanto el partido inaugural como el definitorio), y por el otro un empate en cero (Holanda v. Suecia) y una goleada por tres goles a cero (de Inglaterra a Suiza)

${ }^{9}$ Sin embargo, al no ser los datos relevados por nosotros mismos, no conocemos los criterios específicos que utilizó la empresa Benq, encargada de dicha labor; ¿Consideró el pase a partir de la intención o solo con el criterio de que llegó a otro jugador del mismo equipo? ¿Cómo resolvió el tema de los rebotes fortuitos? ¿Y de los pases con la mano que dio el arquero? Hacia el final de trabajo propondremos un criterio para tomar el pase fundado en la perspectiva de alguno de los técnicos más reconocidos del fútbol mundial, mientras tanto nos manejamos con la hipótesis ad hoc de que Benq utilizó (dado el gran número de pases contados) un criterio poco restrictivo.
} 


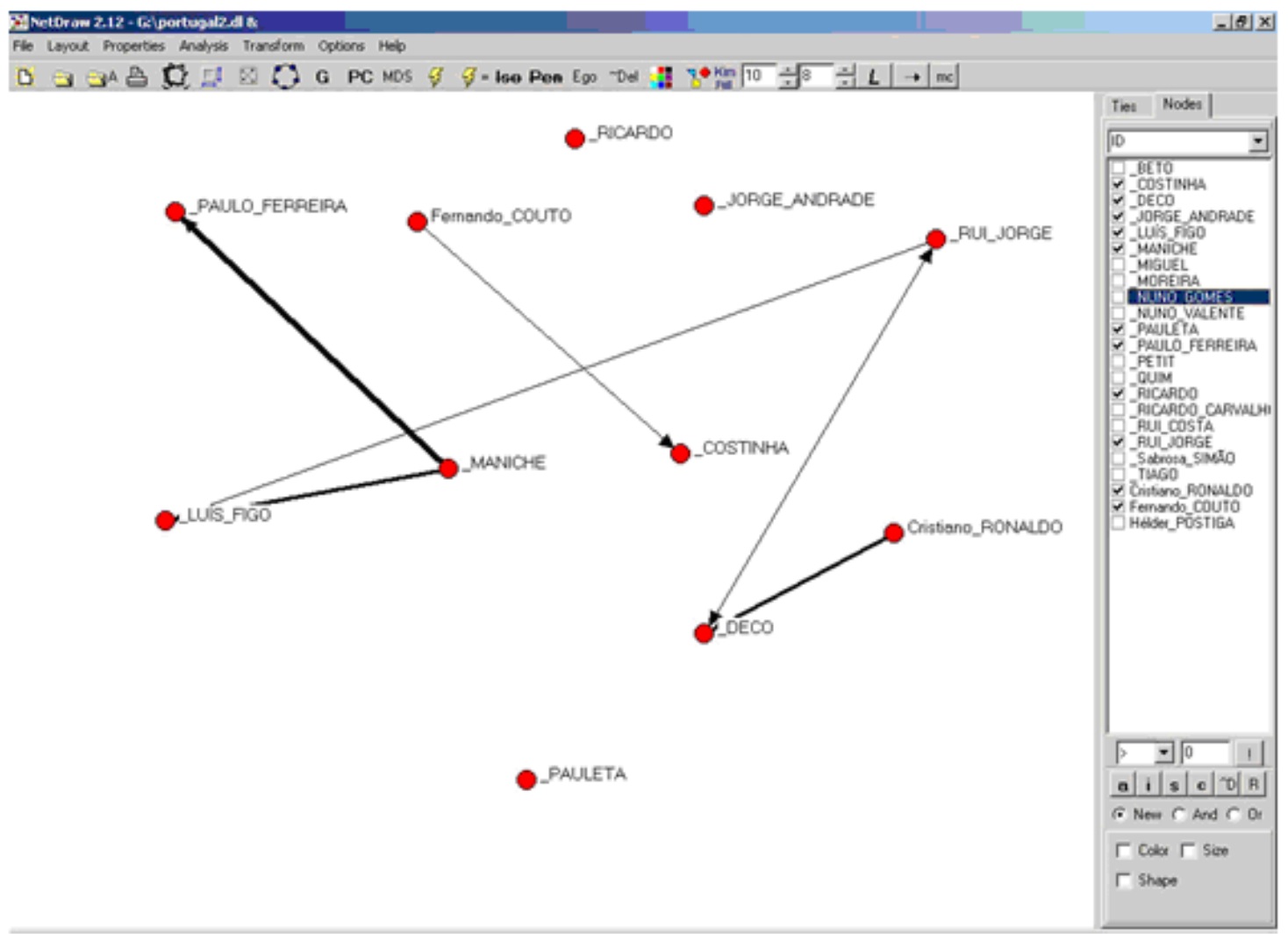

Figura 1: red del equipo de Portugal en el partido final, diagramado con el software NetDraw dentro del paquete UCINET 6

A partir de esto podríamos, por ejemplo, profundizar el análisis de determinadas duplas (díadas) y ver hasta qué punto los jugadores "se buscan" entre sí o realizar estadísticas comparativas teniendo en cuenta el flujo entre dos jugadores clave (el volante ofensivo y el delantero, dos volantes ofensivos, etc) y la cantidad de llegadas que el equipo produce. Una nueva manera de analizar que, por ejemplo, nos podría graficar desde otro lugar la influencia del tandem Gullit-Van Basten en la Holanda de finales de los ochenta (por mencionar una de las tantas duplas que marcaron un equipo), o bien de la tríada Ronaldinho-Messi-Eto`o que tan buenos resultados le trajo al Barcelona en la temporada 2005/2006.

De este modo estaríamos dando vuelta el elegante y original postulado de Mark Granovetter (1973), ya que nuestro análisis busca formalizar la fuerza de los vínculos...fuertes. 


\section{Análisis e interpretación de datos reticulares: Correlaciones entre medidas propias del ARS y conceptos relativos a la táctica y la estrategia en el fútbol}

Usaremos las medidas de grado (degree) ${ }^{10}$, densidad (density), y centro/periferia (core/periphery) ${ }^{11}$ aplicadas a las redes mapeadas usando el software Ucinet ${ }^{12}$. Y a partir de este análisis, discutiremos de qué manera estas medidas podrían contribuir con un aporte sustancial al contenido de la teoría del fútbol, al otorgar una visión alternativa y complementaria de la misma. En todo momento haremos referencia a conocimientos tácticos aportados por los directores técnicos ${ }^{13}$ que fueron consultados y por la bibliografía relativa a lo que podriámos denominar "teoría futbolística" ${ }^{14}$ utilizada. Es fundamental tomar este punto de partida ya que, así como diferentes estructuras de redes tienen diferentes implicaciones para los nodos (2003:9-14), distintos esquemas tácticos distribuyen la pelota de manera diferente, de acuerdo a la táctica específica que el cuerpo técnico determina, los atributos de los jugadores y las circunstancias impuestas por el rival en el desarrollo del partido, ya que, como reza una máxima típica del fútbol, el desempeño de un equipo está condicionado por el desempeño del equipo rival. Además es imprescindible contar con un fuerte apoyo estadístico y realizar un análisis integral que conjugue la dimensión reticular con la estadística y teórica.

\section{Centralidad}

En primer lugar, intentaremos determinar si el nodo con degree máximo varía de acuerdo al esquema táctico. Los grados-fuera y grados-dentro (indegree y outdegree) máximos de cada equipo se muestran en los siguientes gráficos

\footnotetext{
${ }^{10}$ Freeman LC, 1979

${ }^{11}$ Función Corr, Borgatti SP y Everett MG, 1999

12 Borgatti, S.P., M.G. Everett, y L.C. Freeman. 2002. Ucinet 6 for Windows: Software for Social Network Análisis. Harvard: Analytic Technologies

${ }^{13}$ Gonzalo Castro, DT selección de FFyL; Javier Gustavo Bundio, DT inferiores Arsenal de Sarandi.

${ }^{14}$ Nos referimos a los libros de Dante Panzeri: Fútbol, dinámica de lo impensado, y de Luis Zubeldia: Táctica y estrategia en el fútbol.
} 


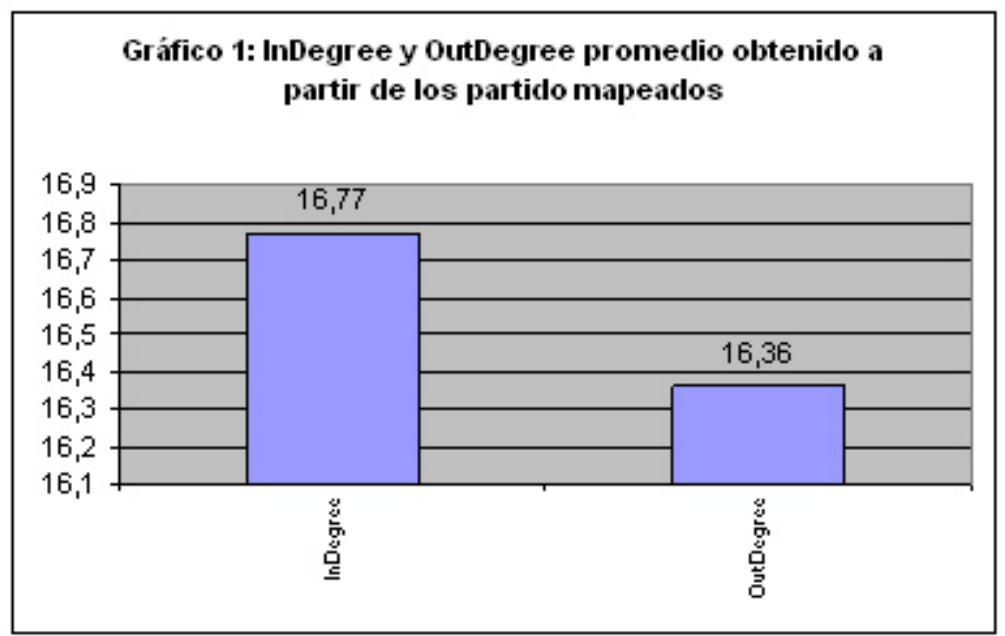

Gráfico 2: Jugadores de mayor OutDegree de cada equipo mapeado

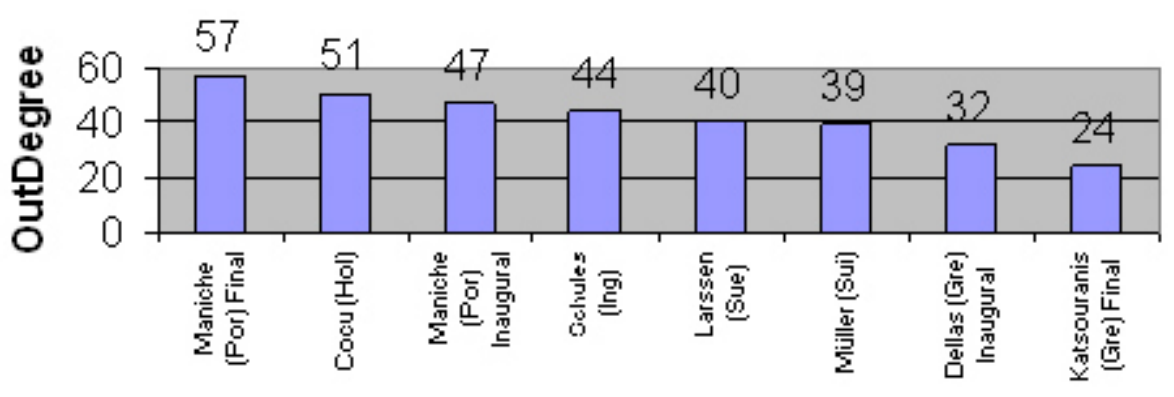

Gráfico 3: Jugadores de mayor InDegree de cada equipo mapeado

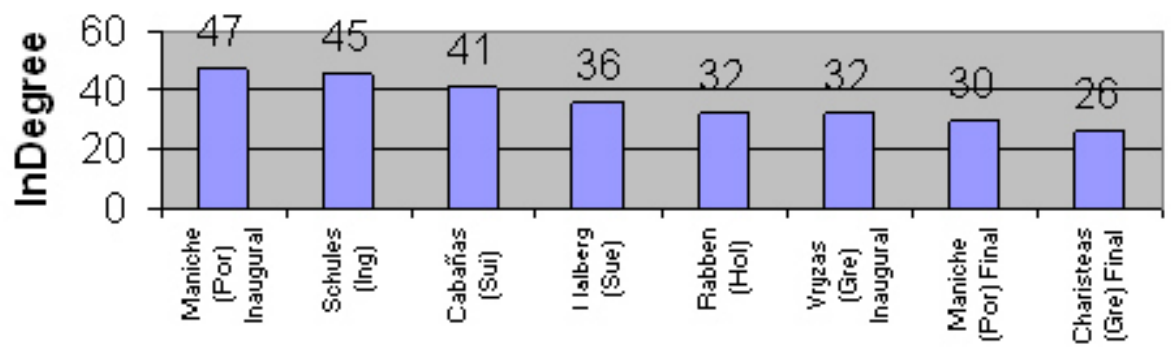


Relacionamos estos datos con la información táctica disponible. En el caso de los esquemas ofensivos (4-3-3, 4-3-1-2 $)^{15}$ el juego pasa por los volantes, son ellos los que crean las variantes ofensivas y distribuyen el balón, por lo que es de esperarse altos degrees en estos jugadores. En el caso de los esquemas defensivos (4-4-2, 45-1), la pelota efectúa un salto por encima de las líneas de volantes, dándose la circulación más entre defensores y delanteros, siendo estos últimos quienes resuelven las oportunidades de gol. Consideramos en este trabajo al grado-dentro como el equivalente reticular de la medida estadística pases recibidos, y al gradofuera como equivalente de pases dados correctamente. Ambos son indicadores de un tipo de juego particular, una constante táctica. A partir de ello se pueden realizar las siguientes observaciones:

a) Los grados máximos de Portugal (Maniche) disminuyeron en la final con respecto al partido inaugural. Hay sin embargo una continuidad táctica porque es el mismo jugador quien los posee;

b) en uno y otro partido, los jugadores griegos más buscados por sus compañeros fueron los delanteros centrales;

c) los jugadores con máximos grados tienden a ser volantes (Maniche para Portugal, Cocu y Robben para Holanda, Scholes para Inglaterra, Cabañas para Suiza), en su mayoría, son los equipos ofensivos los que poseen un volante con alto degree.

Para poder determinar de qué manera varía la centralidad de acuerdo al dibujo táctico, se seleccionaron dos redes realizando el paso metodológico previo a sumar los datos reticulares de los titulares y sus reemplazantes ${ }^{16}$, luego obtuvimos los grados promedio de defensores, volantes y delanteros y comparamos su proporcionalidad relativa. Los gráficos se construirán respetando las posiciones de los jugadores en el esquema táctico (de derecha a izquierda y desde defensa a ataque). ${ }^{17}$

\footnotetext{
${ }^{15}$ La numeración indica el modo en que un equipo para a sus jugadores dentro del campo, por ejemplo: 4-4-3 indica la utilización de cuatro defensores, 4 mediocampistas y 3 delanteros, se omite mencionar al arquero ya que resulta obvia su inclusión como tal atrás de la línea de defensores.

16 Este paso metodológico tiene el objetivo de simplificar el análisis sin descartar la participación de ningún jugador, como sí lo haría establecer un umbral de corte. Sin embargo, existe el problema del cambio posicional que viene luego de una sustitución. En este trabajo consideramos que los jugadores mantienen siempre su posición en el esquema táctico.

17 Por razones de espacio se mostrará solamente gráficamente un esquema ofensivo y uno defensivo correspondientes a la final de la Eurocopa.
} 
Portugal (Final)
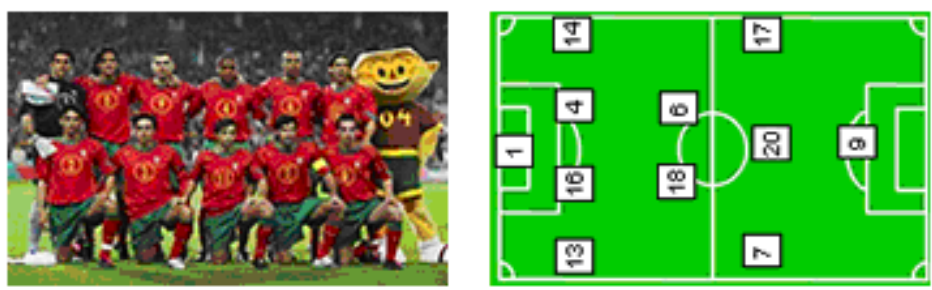

Gráfico 4: InDegrees y OutDegrees de los jugadores de Portugal en la Final de la Eurocopa

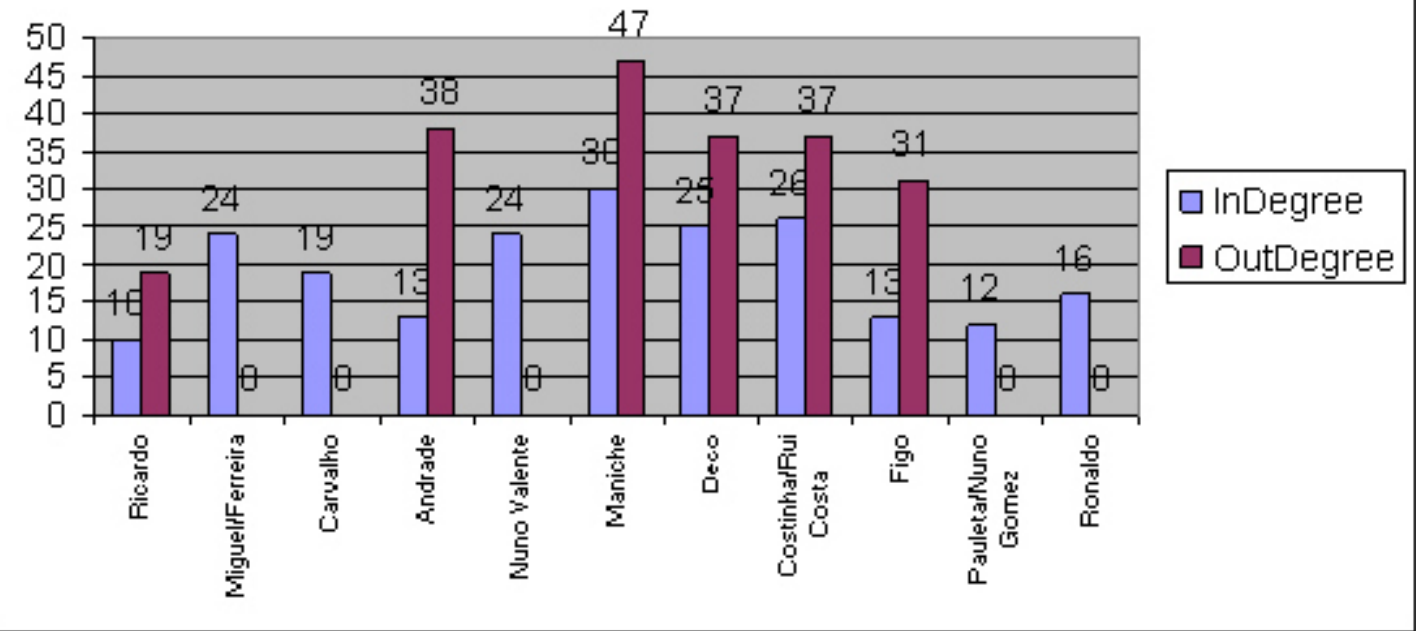

Grecia (Final)
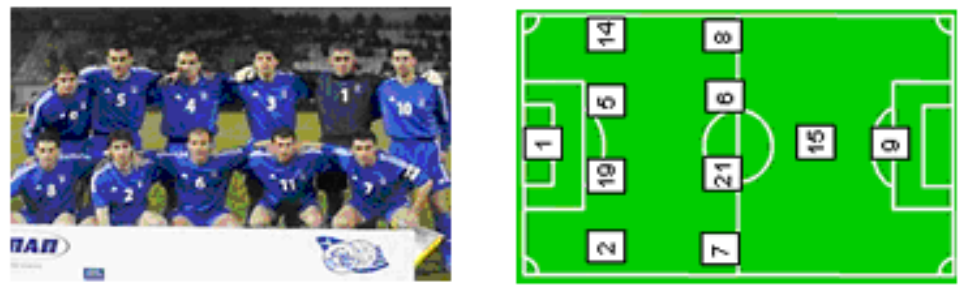

Gráfico 5: InDegrees y OutDegrees de los jugadores de Grecia en la Final de la Eurocopa

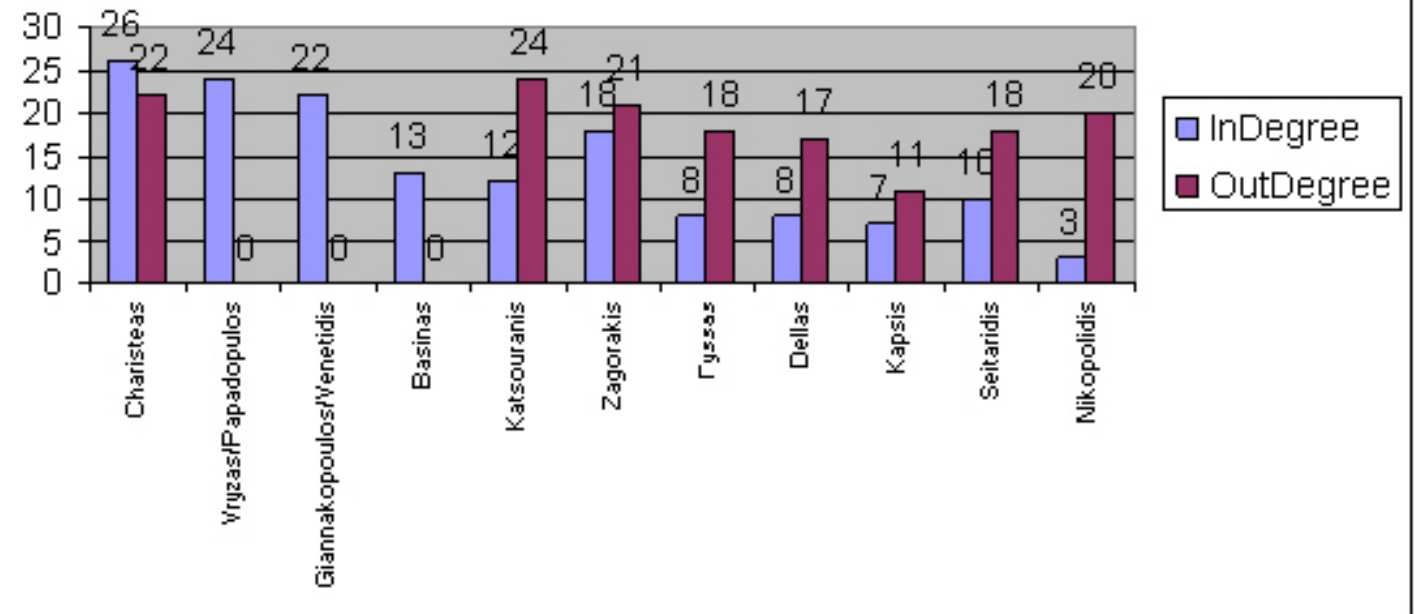


Observando estos datos se presenta la anomalía de grado-fuera $=0$. Consideramos que se debe a un incorrecto relevamiento de datos, si suponemos que el criterio utilizado fue el de pase correcto, pero al no conocerlo no encontramos interpretación a estos datos. De acuerdo a nuestro propio relevamiento de datos durante la Copa América 2007, un grado-fuera nulo resulta altamente improbable aunque no imposible.

A continuación se presenta una tabla comparativa de los promedios de degrees en los cuatro sectores del campo de juego:

\begin{tabular}{|c|c|c|c|c|}
\hline País & Arquero & Defensa & Mediocampo & Delantera \\
\hline & $10 \%$ & $20 \%$ & $27 \%$ & $13.67 \%$ \\
\hline & $3 \%$ & $8.25 \%$ & $16.25 \%$ & $25 \%$ \\
\hline
\end{tabular}

Outegree

\begin{tabular}{|c|c|c|c|c|}
\hline País & Arquero & Defensa & Mediocampo & Delantera \\
\hline & & & & \\
\hline & $19 \%$ & $15,5 \%$ & $40,34 \%$ & $10,34 \%$ \\
\hline $20 \%$ & $20 \%$ & $16 \%$ & $11,25 \%$ & $11 \%$ \\
\hline
\end{tabular}

Tablas 1 y 2: Indegree y Outdegree normalizado correspondientes al partido final

Se desprende de estos datos que Portugal, que hace uso de un esquema táctico ofensivo (4-3-3) presenta un InDegree y OutDegree promedio mayor en el mediocampo. En cambio, el esquema defensivo de Grecia presenta un mayor InDegree promedio en la delantera y un OutDegree promedio mayor en la defensa. Estos datos pueden estar indicando dos estilos de juego diferentes. Portugal parece tener más juego en el mediocampo mientras que en Grecia, el flujo de pelota parece dirigirse desde la defensa al ataque (contraofensiva). 
Para evaluar estas interpretaciones, aplicamos el algoritmo CORR de core/periphery ${ }^{18}$. Los resultados se presentan en el siguiente cuadro:

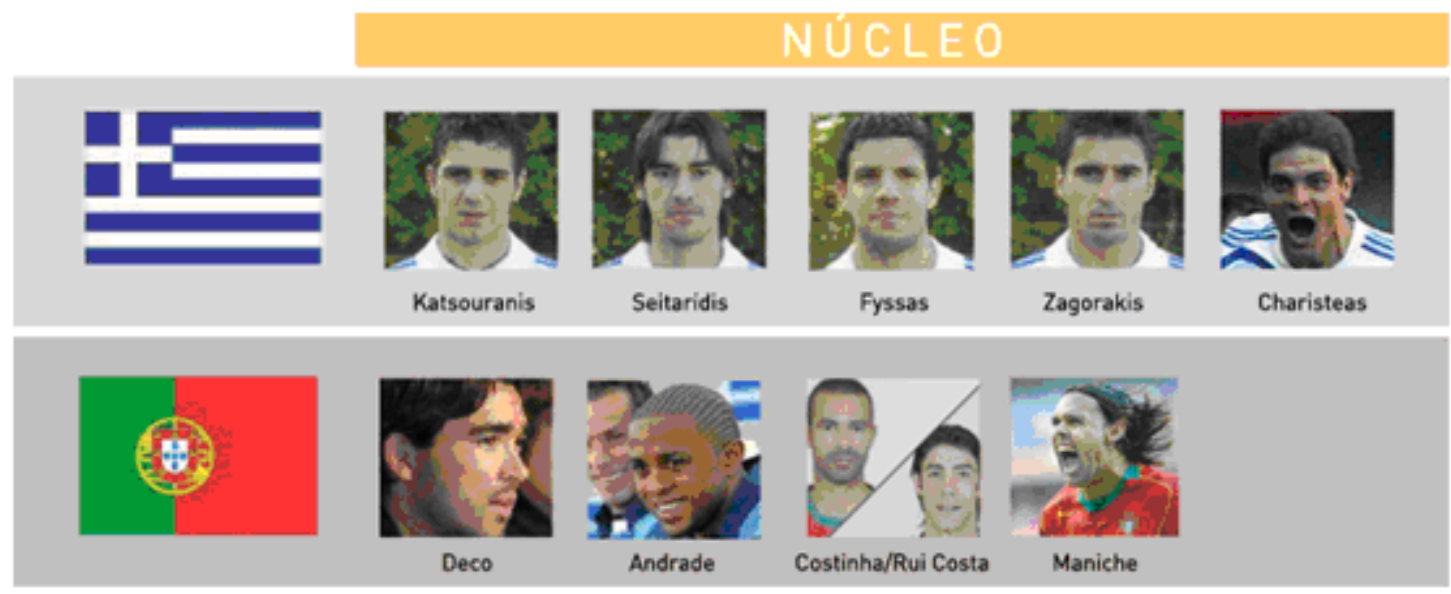

Figura 2: jugadores “núcleo” de Grecia y Portugal según la medida Centro/Periferia del UCINET 6

Estos datos aportan comentarios muy interesantes con respecto al juego llevado a cabo por cada equipo. Para el primer caso, los datos indican una preferencia por la salida lateral (Seitaridis y Fyssas son los dos defensores laterales), por el juego sobre la banda derecha (Seitaridis, Zagorakis y Katsouranis) y por la verticalización apelando al centrodelantero (Charisteas). En el segundo caso, el núcleo de la red es el mediocampo íntegro de Portugal (Maniche, Deco y Costinha/Rui Costa), lo que permite suponer que Portugal apeló al mediocampo como motor del equipo a la hora de generar juego.

\section{Densidad}

Analizaremos ahora la densidad en relación con la táctica del equipo, buscando correlacionar ambos conceptos a partir de mostrar como la densidad varía dependiendo del esquema táctico que se utilize. Para analizar la red se sumaron los datos reticulares de titulares y sus respectivos reemplazos, ya que la entrada de jugadores finalizando el partido, que no participan demasiado en el juego, provoca modificaciones sustanciales en la densidad de la red. Consideramos estas modificaciones de la densidad como no significativas para nuestro estudio en relación con la densidad de la red entendida como "volumen de juego" del equipo, por lo que reducimos la red inicial a una de 11 nodos. El siguiente gráfico muestra una comparación entre todas las densidades:

\footnotetext{
${ }^{18}$ Brevemente recordemos que este cálculo tiene dos costados, el positivo que marca los nodos cuyas relaciones son más fuertes y uno negativo que relaciona a los nodos más distantes unos de otros
} 


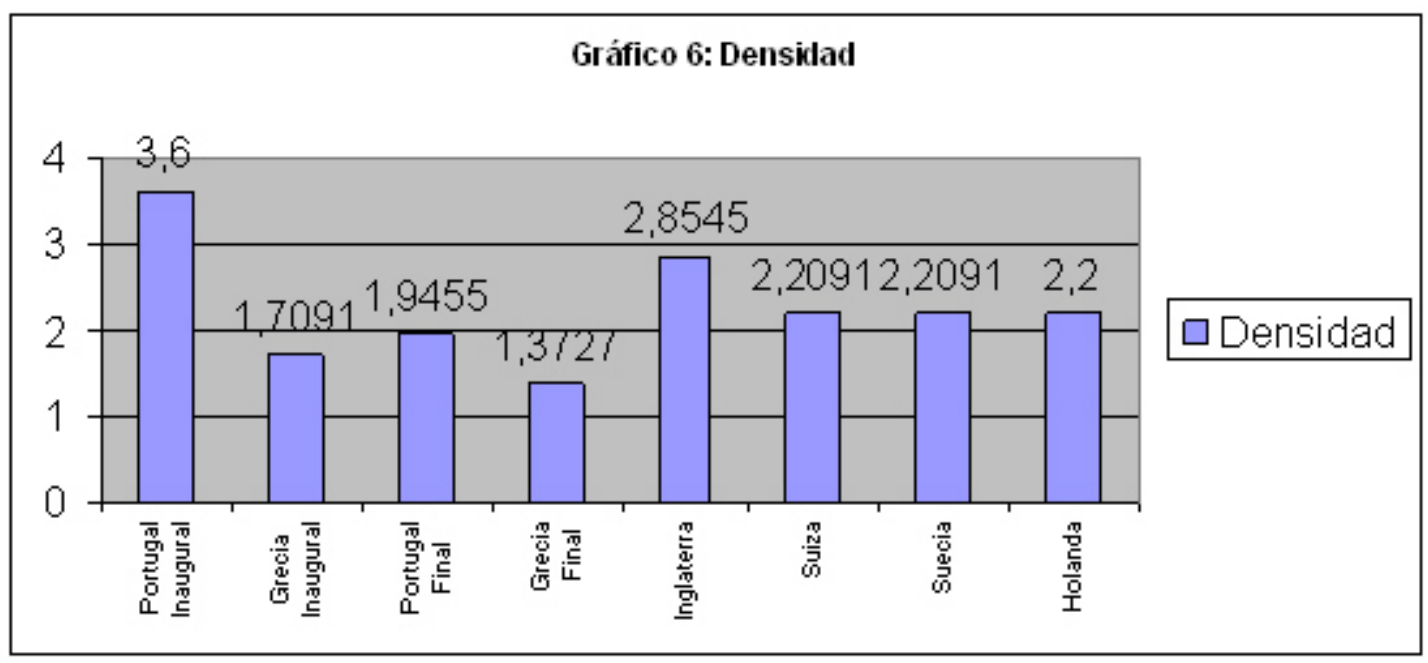

Observamos que no hay relación directa entre los resultados de los partidos y las densidades de las redes, pero sí se aparece una mayor densidad de los equipos con esquemas tácticos ofensivos en el caso de Portugal-Grecia (inaugural y final) e Inglaterra-Suiza. Es tentador relacionar la igualdad de densidades de SueciaHolanda con el empate obtenido, sin embargo se necesitan más datos sobre otros partidos para llegar a conclusiones de mayor peso. Las observaciones más interesantes surgen de la comparación de la densidad con ciertos datos estadísticos, como la posesión y tiros al arco. Este tipo de cotejamiento suministra información sobre el ritmo de juego de un equipo.

El siguiente gráfico muestra la posesión del balón en los partidos de Portugal v. Grecia final y Suecia v. Holanda y un gráfico comparativo de densidades. Como paso metodológico previo se transformaron las densidades en una medida porcentual relativa, sumando las densidades de las redes de cada partido y estableciendo la relación porcentual de cada red en relación con la suma de las densidades. Los resultados figuran en el siguiente gráfico: 


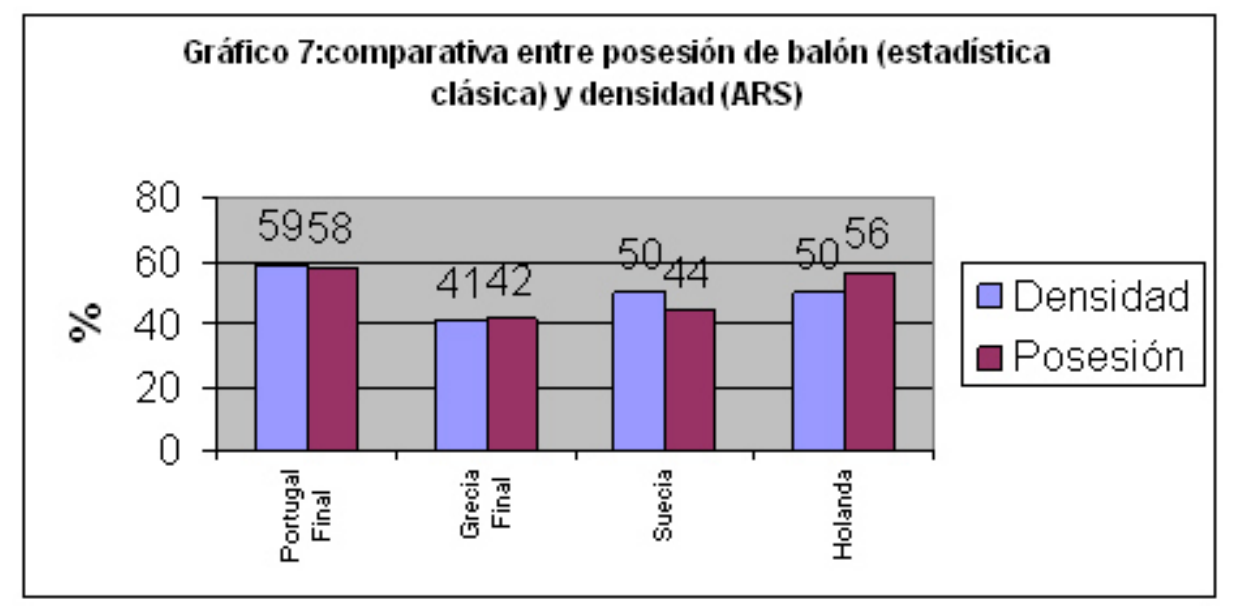

En el caso de la final sí parece haber una relación directa entre posesión y densidad, las relaciones relativas entre las densidades y entre las posesiones son similares, pero en el caso del empate entre Suecia y Holanda la relación entre las densidades no coincide con la posesión del balón. El análisis indica que el caso de Suecia-Holanda puede estar mostrando los desfasajes en el ritmo de juego, Suecia puede haber hecho sus pases mucho más rápido que Holanda (lo que se ejemplifica por la densidad similar y la menor posesión). El análisis permite otra suposición interesante, los jugadores holandeses pueden haber retenido más la pelota que sus adversarios (mayor posesión) y resuelto las jugadas en alternativas que no se reflejan en los datos reticulares, es decir, no se consideran como relación entre nodos: tiros al arco, pérdida de pelota, malos pases o centros (de ahí la igualdad de densidad). Las estadísticas de disparos al arco y corners, como indicadores de ofensividad, avalan esta última hipótesis (aunque no descarta la primera):

\begin{tabular}{|c|c|c|c|c|}
\hline Equipo & Suecia & Holanda & Portugal & Grecia \\
\hline Disparos & 17 & 23 & 17 & 4 \\
\hline Disparos al arco & 4 & 8 & 5 & 1 \\
\hline Saques de esquina & 5 & 11 & 10 & 1 \\
\hline
\end{tabular}

Tabla 3: Estadísticas clásicas referidas a los partidos de Suecia - Holanda y Portugal - Grecia Final

En el caso de Portugal, los datos estadísticos sí se corresponden con lo esperado. Su alta densidad en relación con Grecia, sumado a la mayor posesión de la pelota, indica un mayor control del balón del primero, repercutiendo en el volumen de las situaciones de gol ( El 81 \% de las situaciones de gol fueron para Portugal!).

Realizamos este mismo análisis con otros partidos de la Eurocopa de los que disponíamos del dato posesión de balón y en los cuáles no concordaban este dato y la densidad de la red. Los datos se muestran en la siguiente tabla: 


\begin{tabular}{|c|c|c|c|}
\hline & Posesión & Densidad & Resultado \\
\hline Portugal & 47 & 61,48 & 2 \\
\hline Holanda & 53 & 38,52 & 1 \\
\hline Grecia & 49 & 74,94 & 1 \\
\hline $\begin{array}{c}\text { República } \\
\text { Checa }\end{array}$ & 51 & 25,06 & 0 \\
\hline
\end{tabular}

Tabla 4: comparativa entre las medidas de posesión (estadística clásica), densidad (ARS) y resultado

Los datos de disparos al arco y córners permiten obtener conclusiones similares a las obtenidas con el caso de Holanda y Suecia, ya que indican ofensividad por parte de Portugal en el primer partido. En el caso de Grecia v. República Checa es enigmático, ya que los indicadores de ofensividad están de lado de Checa pero al no disponer del partido mapeado según nuestros criterios no podemos avanzar en una respuesta satisfactoria a las hipótesis planteadas. Lo que si se determina es que no siempre coincide la densidad con la posesión de balón, lo que muestra que ambas medidas indican cosas diferentes y la posibilidad de tomar esta diferencia abre nuevas perspectivas de análisis.

\section{Densidades y proveniencias}

Utilizamos el cálculo de densidad para proyectar otro análisis fundado en el ARS en el que también encontramos un interesante correlato con una estadística propia del fútbol. El mismo surge a partir de la hipótesis de que los jugadores pertenecientes a un mismo club, es decir, aquellos que son compañeros todo el año, se pasan más la pelota entre ellos que aquellos que provienen de clubes disímiles y sólo juegan juntos en el seleccionado. Un tema que en sus diferentes variantes (no solo club de proveniencia, sino también jugadores que son más amigos entre sí o que llevan más tiempo en el club, entre otras), ha desatado múltiples polémicas a lo largo de la historia del fútbol. Seguramente algún fanático de este deporte recuerde, por ejemplo, el altercado que mantuvieron algunos de los jugadores de la selección holandesa antes del mundial de 1998 disputado en Francia, cuando algunas declaraciones cruzadas dejaban entrever problemas de tintes racistas en el seno del plantel. Incluso buena parte del periodismo insinuaba que los jugadores de tez morena (Seedorf, Davids, Winter y Kluviert entre otros, varios de ellos provenientes de colonias holandesas en América del Sur, como Surinam) no le pasaban la pelota a los de tez blanca (Bergkamp, los hermanos De Boer, Overmars, y otros) y viceversa. Holanda hizo un buen papel en aquella Copa del Mundo y el tema quedó en el olvido, pero es aquí retomado como ejemplificación de la dirección de nuestro desarrollo. 
Pero volvamos a la Eurocopa, ya que a fines expositorios encontramos en Portugal un caso muy interesante para desarrollar una aproximación como la propuesta, ya que la mitad de su equipo base eran jugadores provenientes del Porto FC de aquel país, equipo que en el año 2004 se había proclamado nada campeón de Europa ${ }^{19}$. En efecto, a partir de dividir la red correspondiente al partido final, confeccionamos dos matrices diferentes. La primera, con aquellos jugadores (y los lazos entre ellos) que habían jugado en aquel Porto y la segunda, de iguales características pero con aquellos futbolistas que llegaron desde diferentes clubes:
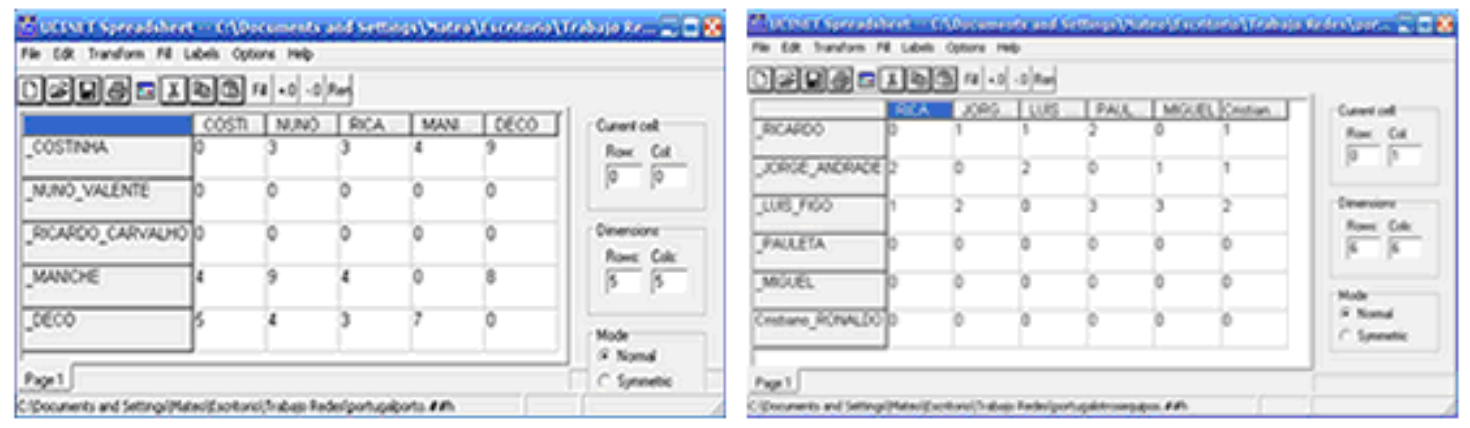

Figura 3: Matrices correspondientes a los jugadores portugueses cedidos a la selección por el Porto FC y del resto de los jugadores del seleccionado

Aplicamos los respectivos cálculos de densidad a cada una de las matrices y obtuvimos los resultados de infografía presentado a continuación:

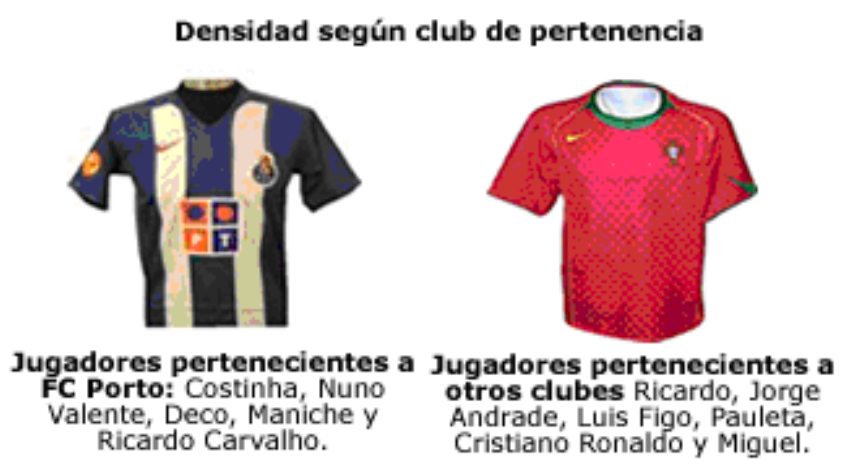

Densidad: 3,150

Densidad: 0,7333

Figura 4: Densidades tomadas a partir de la división de la matriz original en dos nuevas matrices, una agrupando a los jugadores que se desempeñaban en el Porto FC, y la otra agrupando al resto de los jugadores

Si bien los resultados obtenidos a partir del análisis de las densidades correspondientes al mostrar una marcada diferencia de densidades parece confirmar nuestra hipótesis, es necesario remarcar que se trata de una conclusión

${ }^{19}$ Ver detalle en http://en.wikipedia.org/wiki/UEFA_Champions_Leaque_2003-04 
por lo menos apriorística, ya que se debe tener en cuenta que los jugadores del Porto no sólo tienen ese atributo, sino que además juegan en sectores del campo donde el flujo de pases es mayor, están dotados técnicamente y su función principal dentro de la táctica del equipo es (especialmente en los casos de Costinha, Deco y Maniche), hacer una buena distribución de balón, algo que no se espera de futbolistas como Pauleta (un delantero de área cuya especialidad es rematar las jugadas) o Ricardo (el arquero del equipo) que se encuentran en el otro grupo.

Por otro lado, los resultados que muestra el cálculo de centro/periferia (ver cuadro 1) aportan a los fines de este desarrollo particular, ya que mediante otra vía nos muestran una mayor interrelación entre los jugadores provenientes del Porto, incluyendo 4 de 5 (y siendo el quinto Rui Costa, un jugador que entra desde el banco de suplentes para reemplazar a Costinha del Porto) dentro del centro y relegando sólo uno a la periferia de la red. Y aunque ninguno de estos cálculos nos permita concluir efectivamente en favor de nuestra hipótesis, creemos que ambos aparecen como herramientas interesantes para una posterior profundización, que posiblemente deba involucrar datos no reticulares. En otras palabras, estos cálculos no han resuelto el caso pero suman antecedentes de potencial valor definitorio.

Retomando líneas más generales, queda claro que más allá de estos resultados no se puede establecer una conclusión definitiva, entre otras cuestiones, por la escasez de redes mapeadas hasta el momento. En lo que sí podemos enfatizar, es en la necesidad de complementar el análisis de redes aplicado al deporte con datos estadísticos individuales y grupales propios del mismo.

A partir de lo expuesto es podemos concluir la importancia de complementar el ARS aplicado al fútbol con datos estadísticos. Entendemos, a partir de las múltiples posibilidades que surgen de dicha conjunción, que este es uno de los caminos fundamentales a seguir en búsqueda de conclusiones superadoras. Futuros trabajos podrían demostrar la no correspondencia directa entre densidad y posesión, indicando que las redes pueden tener un ritmo diferente. Esto abriría el análisis a la introducción de la diacronía en el ARS aplicado al deporte en la forma de "ritmo de juego". Por otro lado, se podrían comprobar con mayor detalle ciertas hipótesis que surgen de este trabajo. Entre otras posibilidades, se podría establecer una proporción entre los grados promedios de defensas, volantes y delanteros como un atributo de ciertos esquemas tácticos, o bien, desarrollar la posibilidad de entender el grado como "volumen de juego", con las herramientas centro/periferia y Ncamarillas ( $\mathrm{N}$-cliques) como recursos para observar los posibles ejes sobre los que se articula la estrategia de un equipo a partir de su mayor interconexión. 


\section{El lado oscuro del análisis de redes futbolísticas}

EI ARS aplicado al fútbol presenta problemas de orden de relevamiento de datos muy importantes. El volumen de pases durante un partido potencia la dificultad de contabilizar los datos reticulares. En este sentido resultaría de utilidad particionar el partido y relevar los datos durante períodos determinados. Además, se necesita realizar múltiples mapeos relacionados con los cambios posicionales de jugadores o aquellos que se producen a partir de disposiciones estratégicas. Los cambios posicionales introducen el problema de la definición y correlación entre el flujo de pelota y el esquema táctico. Cada esquema táctico presenta una diferente circulación de pelota y alternativas de juego. En el mejor de los casos el flujo de pelota es condición necesaria pero no suficiente para indicar si un equipo es defensivo u ofensivo. Observamos que la densidad y el grado promedio de volantes con respecto a defensas y delanteros y las densidades de ciertas zonas de la red pueden ser buenos indicadores de ofensividad. Sin embargo, el esquema táctico es un dato que se dispone y no se deduce de ningún indicador, estos sirven como tales si se conoce de antemano la formación del equipo.

Con respecto a este punto, estamos preparando un análisis del desempeño de la Argentina en la Copa América de Venezuela 2007, realizando el relevamiento de datos por nuestra cuenta, usando como criterio para determinar la existencia del lazo el de pase correcto, esto es pase que, enviado por un jugador del equipo " $X$ " es recibido por otro del mismo equipo. Desde el inicio del torneo tuvimos que superar el obstáculo que supone idear un sistema para relevar los datos sin hacer engorrosa la tarea. De este modo buscamos realizar una investigación propia de redes sociales desde el inicio de la misma (2007b).

En este sentido la técnica desarrollada es un aporte para todos los analistas de redes interesados en este tipo de análisis. La técnica mencionada la llamamos mapeo de cadena de pases. Suponiendo el siguiente ejemplo: el futbolista Verón le quita el balón a un contrario y se la pasa a Messi, éste se la devuelve de primera, Verón domina la pelota y lanza un balón largo que se va fuera de los límites del campo. La cadena toma la forma de Verón-Messi-Verón. Posteriormente esta información se vuelca en una matriz de adyacencia que queda parcialmente ${ }^{20}$ de este modo:

\footnotetext{
20 La matriz presentada a modo de ejemplo, representa una única situación dentro del match y supone que la información se vuelca inmediatamente. En términos más cercanos a las posibilidades reales del investigador a cargo del relevamiento, su trabajo incluye, en primer lugar la confección de todas las
} 


\begin{tabular}{|c|c|c|}
\hline & Verón & Messi \\
\hline Verón & - & 1 \\
\hline Messi & 1 & - \\
\hline
\end{tabular}

Tabla 5: Matriz que resume los datos reticulares de la cadena de pases

Este sistema permite abreviar el proceso de relevamiento de datos, ya que los mismos pueden ser tomados en "tiempo real", es decir, a medida que se desarrolla el encuentro. En un segundo momento se vuelcan estos datos a una matriz de adyacencia que incluye la totalidad de los jugadores que participaron del partido.

\section{¿Hacia una nueva estadística? Perspectivas y avances en las la aplicación del ARS al fútbol}

A pesar de las dificultades expuestas, se esperan importantes avances en la implementación del ARS al fútbol. Estos están supeditados al relevamiento y mapeo de más partidos, tarea para la cual creemos estar aportando herramientas fundamentales. Se debe contar con un fuerte apoyo estadístico y teóricoperiodístico para poder establecer las generalizaciones que se consideran imprescindibles como objetivos del análisis. En este sentido esperamos que el mapeo y análisis de los partidos de la selección Argentina durante la Copa América ${ }^{21}$ aporte información que posibilite un avance más definido en este tipo de análisis. En otro orden de cosas, esperamos una futura implementación del ARS en otros deportes, entendiendo que la suma de posibilidades en este sentido permitiría establecer este enfoque como una herramienta imprescindible para todo analista deportivo.

Para concluir, las posibles implementaciones son de tres órdenes. En primer lugar se plantea al ARS como alternativa y complemento del análisis estadísticoperiodístico. Es sintomático del origen relativamente reciente del análisis de redes que no se encuentren trabajos de este estilo. Se considera que es la manera más intuitiva de presentar los datos de un partido al público en general que cualquier resumen estadístico o análisis periodístico. En segundo lugar, el ARS puede contribuir considerablemente a la teoría del fútbol y servir como validador o

cadenas de pases que se dan durante el partido y luego la depuración de los datos a partir de su traslación a la matriz de adyacencia correspondiente.

${ }^{21}$ Bundio, J. Y Conde, M. Nuevas exploraciones en fútbol y redes sociales: Un análisis de la Copa América 2007. En preparación. 
falsador del sentido común involucrado en el deporte ${ }^{22}$. En tercer lugar, es una herramienta de gran utilidad para el asesoramiento del cuerpo técnico, ya que, entre otras funciones, permite efectivizar la presentación de datos en la charla técnica, posibilita una nueva forma de evaluación del rival y de análisis de partidos, da lugar a la construcción de base de datos más extensas y completas que las actuales, e incluso da lugar al análisis del desempeño individual en la red total, la evaluación del rendimiento por sectores, o la comparación de la distribución de pelota en entrenamiento y en los partidos de campeonato, por lo que viabiliza la evolución detallada del desempeño táctico de una formación. Entendemos que estamos frente a una herramienta, que de poder desarrollarla, es más que efectiva para brindar consultoría a los entrenadores, especialmente a aquellos más apegados a dinámicas formales de trabajo, como los ex seleccionadores argentinos Marcelo Bielsa y Carlos Bilardo, el español Rafa Benitez (que condujo al Liverpool a ganar la Champions League en el 2005), o el propio Fabio Capello (que terminó su ciclo en el Real Madrid con un nuevo título de Liga para los blancos en la pasada temporada). Se espera que este trabajo aporte un conocimiento sustancial en pos de estos objetivos.

\section{Conclusiones: El análisis de redes sociales como herramienta para una ponderación del juego}

Intentamos a través del presente trabajo desarrollar una serie de posibilidades metodológicas que desde nuestro punto de vista nos aproximan a un nuevo nivel de análisis del fútbol a partir de los aportes provenientes del ARS. No obstante, entendemos que las posibilidades de esta conjunción no se agotan allí.

En este sentido comprendemos que el fútbol puede nutrirse del despliegue analítico del fútbol, y el ARS de la difusión y practicidad propia del ambiente futbolistíco. En reiteradas ocasiones nos encontramos ante estudiantes que desechan este tipo de perspectivas por considerarlas demasiado difíciles de entender o directamente por desconocer sus mecanismos y posibles objetos de estudio. A modo de aporte para superar esta cuestión, estamos preparando un material ${ }^{23}$ de fácil lectura donde se presentan los conceptos básicos del ARS en analogía a conceptos futbolísticos típicos. Aspiramos a que en un país donde el fútbol esta tan extendido como la

\footnotetext{
${ }^{22}$ En el sentido de que formaliza algunas de las frases hechas del fútbol como "Hay que tapar al volante central porque es la salida del equipo", o bien "la columna del team la forman el 2, el 5, el 10 y el 9 de área".

23 Bundio, Javier y Conde, Matías: “Algunos conceptos básicos de redes sociales: una analogía futbolística". En preparación.
} 
Argentina, un trabajo de este tipo pueda resultar un ejercicio útil para quienes enseñan la ciencia de las redes, es decir, que resulte un aporte al campo de la didáctica del ARS y se promueva como una posible carta de presentación del enfoque en niveles de educación secundarios y terciarios.

Además de las razones metodológicas y didácticas, adherimos a los que sostienen que todos los trabajos que se presentan tienen un costado ideológico, por lo que, el aquí expuesto no puede ser una excepción. Consideramos necesarios desarrollos de este tipo para que se vuelvan a centrar las discusiones sobre fútbol en lo que pasa dentro del terreno de juego y no en toda la parafernalia de los alrededores.

Creemos firmemente que la sobreexposición del hincha por parte de los medios (la referencia es al caso argentino en particular) lo ha colocado en un lugar que no sólo no le corresponde, sino que le queda grande: el centro del show. La televisión argentina se inundó de programas dedicados a "repasar el folklore del fútbol" que en realidad no hacen más que cederle el micrófono a algún fanático para que este desarrolle un discurso de insultos y amenazas (que lamentablemente suelen no quedar en palabras) a la parcialidad rival. Un verdadero circo donde todas las exageraciones son premiadas; se mistifican líderes mafiosos de barras bravas (ultras) que hicieron y hacen muchísimo por dañar las economías de cada club, se transmiten partidos enteros enfocando únicamente a las hinchadas y luego se les pide a los hinchas que no adquieran un protagonismo que no les corresponde, para peor, se aprovechan los desmanes habituales para bajar un discurso de mano dura por algún otro canal del multimedio y hasta se presentan resúmenes (¡En los programas de fútbol!) de diez minutos del juego contra no menos de quince de la llegada de los hinchas a la estadio (filmada en alta calidad, con música acorde y editada como si fuera una pequeña película) y entrevistas a los jugadores hablando de cualquier cosa menos de fútbol. En efecto, entendemos que esta situación propició y propicia el aumento de los niveles de violencia que se han dado en los últimos años en los estadios de Argentina, donde la agresividad a llegado a límites insoportables y donde indigna, pero ya no sorprende, que los partidos calientes (los definitorios, los clásicos, etc.) terminen con algún muerto y centenares de heridos y detenidos. 
En este marco de fanáticos exacerbados por los medios propulsamos una vuelta al análisis del juego, donde los espacios centrales de los programas, diarios y suplementos deportivos estén dedicados a lo que sucede dentro y no fuera de los límites del campo. Si nos ocupamos cada vez menos de los movimientos de los futbolistas en la cancha y cada vez más el accionar de hinchas, intermediarios y managers, no debemos sorprendernos por este fútbol asustado y torpemente resultadista que se juega en estos días, ni por el crecimiento de las corporaciones y las mafias dentro de este deporte.

Desde ya que no pretendemos haber encontrado, a partir de las aplicaciones propuestas del ARS, ni la solución a la violencia en el fútbol, ni a la mala calidad del juego en los últimos años, pero sí creemos estar aportando desde nuestro lugar, hacia una futura reconfiguración de intereses en lo que al análisis de este deporte respecta.

\section{Bibliografía}

Borgatti, S.P., Everett, M.G. and Freeman, L.C. (2002-2007). Ucinet for Windows: Software for Social Network Analysis. Harvard, MA: Analytic Technologies.

Borgatti, S. (2003). "Conceptos básicos de Redes Sociales" XXIII conferencia Internacional de Análisis de Redes Sociales en Cancún, México. 2003. En http://www. analytictech.com/networks (fecha de consulta: Junio 2007)

Grupo Antropocaos (2007). Exploraciones en Antropología y Complejidad. Editorial S.B., Buenos Aires. 2007.

Granovetter, M. (1973). "La fuerza de los vínculos débiles". Johns Hopkins University. Trad. Maria Angeles Garcia Verdasco.

Hanemann, R. (2000). "Introducción a los métodos del análisis de redes sociales" Dpto. de Sociologia de la Universidad de California Riverside. Versión electrónica disponible en http://wizard.ucr.edu/ rhannema/networks/text/textindex.html (Fecha de consulta: Octubre de 2006)

Jegoo Lee, Stephen P. Borgatti, Jose Luis Molina, Juan J. Merelo Guervos (2005). "Who Passes To Whom: Analysis Of Optimal Network Structure In Soccer Matches", XXV International Sunbelt Social Network Conference, Redondo Beach, CA (EUA), February 19, 2005. http://www. socsci.uci. edu/ ssnconf/conf/masterschedule.html.

Miceli, J. (2007b). "Problemas de validez en el ARS". En Impresión.

Panzeri, D. Fútbol (1964). Dinámica de lo impensado. Buenos Aires: Paidós.

Rivoir, Ana Laura (1999). "Redes Sociales: ¿Instrumento metodológico o categoría sociológica?" en Revista de Ciencias Sociales. Versión electrónica disponible en http://www.lasociedadcivil.org/uploads/ciberteca/articulo_redes.pdf (Fecha de consulta: Marzo de 2007). 
V/A "Juego Redes contra Redes", en Revista REDES. Disponible en http://revistaredes.rediris. es/webredes/eurocopa/datos.htm (Fecha de consulta: Junio de 2007).

Zubeldía, Osvaldo J. (1965). Táctica y estrategia del fútbol. Buenos Aires: Jorge Álvarez. 